\title{
DOI 10.35381/cm.v6i10.141
}

\section{La estructura social en el rendimiento académico de los nuevos ingresos al sector universitario}

\section{The social structure in the academic performance of the new income to the university sector}

\author{
Gabriela Esperanza Zambrano Vera \\ gezambrano@utm.edu.ec \\ Universidad Técnica de Manabí \\ Ecuador. \\ https://orcid.org/0000-0002-5201-2148 \\ María Antonieta Palma Cedeño \\ mapalma@utm.edu.ec \\ Instituto de Lenguas Modernas \\ Ecuador \\ https://orcid.org/0000-0003-3933-0536 \\ Silvia Monserrate Suástegui Solórzano \\ ssuastegui@utm.edu.ec \\ Universidad Técnica de Manabí \\ Ecuador \\ https://orcid.org/0000-0003-2883-8748
}

Recibido: 5 de mayo de 2019

Aprobado: 13 de junio 2019

\begin{abstract}
RESUMEN
El presente estudio tuvo como objetivo analizar la estructura social en el rendimiento académico de los nuevos ingresos al sector universitario. El propósito de la investigación fue analizar los factores asociados al rendimiento académico de los estudiantes universitarios de nuevo ingreso. La metodologia que siguio la investigación fue de tipo descriptiva y de campo con un de tipo no experimental y transversal. Las unidades de análisis fueron los estudiantes que ingresaron durante los lapsos
\end{abstract}


académicos del primer período de 2018. Se utilizó como técnica la encuesta para la recolección de datos, y como instrumento un cuestionario. El análisis permitió concluir que los factores estudiados representan puntos débiles y fortalezas en el rendimiento académico de los estudiantes de recién ingresos, todo si consideramos que el rendimiento académico no sólo se da en términos de calificaciones numéricas, sino su sentir con la realidad universitaria.

Descriptores: Estructura social; Rendimiento académico; Estudiantes universitarios; Factores del rendimiento Académico; Factores contextuales; Factores personales.

\begin{abstract}
This study aimed to analyze the social structure in the academic performance of new income to the university sector. The purpose of the research was to analyze the factors associated with the academic performance of new-entry college students. The methodology that followed the research was descriptive and field type with a nonexperimental and transversal type. The units of analysis were the students who entered during the academic lapses of the first period of 2018. The survey for data collection was used as a technique, and as an instrument a questionnaire. The analysis concluded that the factors studied represent weaknesses and strengths in the academic performance of under-income students, all if we consider that academic performance is not only given in terms of numerical qualifications, but its feeling with university reality.
\end{abstract}

Descriptors: Social structure; Acdemic performance; University students; Academic performance factors; Contextual factors; Personal factors.

\title{
INTRODUCCIÓN
}

El problema del acceso y la permanencia de los estudiantes logrando niveles de calidad adecuados, constituye hoy un elemento de primer orden en el ámbito de la educación superior a nivel nacional y mundial (Ezcurra y col, 2011). Al respecto, desde el pasado siglo, esta problemática ha estado en el centro de los debates académicos como tema recurrente, destacando que la equidad educativa en la educación superior está fuertemente relacionada con todos aquellos factores que inciden en el acceso de la población estudiantil a los estudios universitarios; en este sentido, quizás el más importante se refiere las asociadas a criterios de calidad educativa, donde la cantidad de 
estudiantes que se admiten y los criterios de selección se tornan en condicionantes de la equidad educativa. También, repercuten todos aquellos servicios estudiantiles dirigidos a las poblaciones estudiantiles que aseguren la permanencia y conclusión de sus estudios (Silva, 2010).

Sin embargo, actualmente se han abandonado los enfoques reduccionistas que intentan analizar el rendimiento académico desde una variable única. Existe un generalizado consenso, enmarcado en el paradigma de la complejidad, acerca de que el rendimiento académico de los estudiantes está sobre determinado por múltiples factores interrelacionados. En este sentido, cabe destacar lo propuesto por Garbanzo Vargas (2007), quien sostiene la existencia de diferentes aspectos asociados al rendimiento académico, tanto internos como externos al individuo y los agrupa en factores de orden social, cognitivo y emocional, y los clasifica en tres determinantes: sociales, personales e institucionales.

Cabe destacar, que los estudios del rendimiento académico en la educación superior parecen ser en la coyuntura mundial actual aún más valiosos, debido al dinamismo que experimenta el sector universitario en el marco de una sociedad caracterizada por el rápido avance del conocimiento, la fluidez en la transmisión de la información y los cambios acelerados en las estructuras sociales, en todos los ámbitos, sociales, económicos, culturales, científicos, políticos y tecnológicos, a nivel mundial.

Por otro lado, al tratarse de nuevos ingresos a las casas de estudios superiores, en su mayoría se trata de jóvenes adolescentes, que les toca enfrentarse a grandes retos en su vida universitaria, el compromiso con ellos mismos de rendir en términos de producto, adaptarse a los cambios académicos, compartir su vida académica con un grupo de compañeros, con sistemas de evaluación más fuertes que en la primaria superior, lidiar con sus procesos afectivos, sus maneras de afrontar las circunstancias, entre tantos procesos que vive diariamente el estudiante universitario. A ello, habría que agregarles los factores personales caracterizados al género, edad y demás variables demográficas sumada a la competencia cognitiva, definida como la 
autoevaluación de la propia capacidad del individuo para cumplir una determinada tarea cognitiva, su percepción sobre su capacidad y habilidades intelectuales. Relacionada con la influencia ejercida en el entorno familiar, incide en distintas variables que se asocian con el éxito académico tales como: la persistencia, el deseo del éxito, expectativas académicas del individuo y la motivación. Atendiendo esta situación problemática, este artículo se enfocó en analizar la estructura social en el rendimiento académico de los nuevos ingresos al sector universitario.

\section{DESARROLLO}

\section{Rendimiento académico}

Los estudios del rendimiento académico en la enseñanza universitaria parecen ser en la coyuntura mundial actual de un gran valor, debido a la dinámica que experimenta dicho sector en el marco de una sociedad que se caracteriza por el rápido avance del conocimiento, la fluidez en la transmisión de la información y los cambios acelerados en las estructuras sociales. De manera que hablar de rendimiento académico, refiere en términos generales, conceptos como rendimiento académico, desempeño académico, logros académicos, éxito o fracaso académico, entre otros terminaos, en la mayoría de los casos son utilizados de forma simultanea o modo equivalente, entendiendo que están estrechamente asociados. Desde la perspectiva de la educación superior, el rendimiento o resultados académicos según Luque y Sequi (2002), al momento de proponer su índice de Rendimiento General para la Enseñanza Superior, distinguen dos tipos de rendimiento académico de un alumno, uno de tipo parcial y un segundo de tipo general. El primero, correspondería a los rendimientos parciales alcanzados por el estudiante en cada una de sus actividades académicas; es decir, la regularización y la aprobación de las asignaturas correspondientes a la carrera en la secuencia temporal prevista por el Plan de Estudio, así como al nivel proporcional de logro cognitivo alcanzado en cada materia aprobada. 
El rendimiento académico, también puede ser definido como el conjunto de resultados efectivos obtenidos por el estudiante en determinadas actividades académicas, como respuesta a un proceso de formación interpretable de acuerdo con objetivos 0 propósitos educativos previamente fijados (Velásquez y col, 2008). Por su parte, Castellano y col (2011), lo define como el proceso técnico pedagógico que juzga los logros de acuerdo a objetivos de aprendizaje previstos, el rendimiento a juicio de Maya, Aldana Zavala \& Isea Argüelles (2019), debe ser modelado desde una gerencia educativa con visión transformadora para que este pueda promover la calidad de la institución educativa, por otro lado, Romero \& Villasmil (2018), destacan que el rendimiento académico en el sector universitario, tiene relación con la madurez vocacional de los estudiantes.

\section{Factores asociados al rendimiento académico en la educación superior}

Analizar los factores que influyen en el rendimiento académico de los estudiantes, permite diagnosticar el estado en que se encuentran, conocer sus competencias, sus deficiencias, sus carencias tanto de conocimientos, como culturales, y ejercer desde allí una acción preventiva que luche contra el fracaso en la universidad. En este sentido Artunduaga (2008), clasifica el rendimiento académico en factores contextuales y personales. El primer grupo contiene variables socioculturales, institucionales y pedagógicas. El segundo, incluye variables demográficas, cognoscitivas y actitudinales.

\section{Factores contextuales asociados al rendimiento académico}

En este factor se agrupan todas las variables que estudian aspectos relacionados con el nivel socioeconómico y cultural del estudiante, así como las variables de tipo institucional y pedagógico. Entre las que se distinguen las variables socioculturales relacionadas con el origen sociocultural, Nivel educativo del padre y de la madre, clima educativo familiar, Integración social del estudiante y las variables pedagógicas a 
través de las expectativas y actitudes del docente, la formación y experiencia del docente, la personalidad del docente, el proceso, el proceso didáctico, el acompañamiento pedagógico, el tamaño del grupo y el clima de la clase

\section{Factores personales asociados al rendimiento académico}

Asimismo, Tejedor (2008), asocia el rendimiento estudiantil a variables contextualizadas, de proceso y de producto, tal como se especifican a continuación:

Dentro de estos factores destaca el autor las variables demográficas de Sexo, edad, Estado civil, experiencia laboral y financiación estudios. En relación a las variables cognoscitivas mencionan las aptitudes intelectuales, rendimiento académico previo, capacidades y habilidades básicas, estilos cognitivos y motivación. Finalmente contempla las variables actitudinales de responsabilidad hacia el aprendizaje, satisfacción, interés por los estudios, decisión ante los estudios, planeación del futuro, autoconcepto y habilidades sociales.(Chaves, 2006).

En relación a las variables de contexto, el autor indica las características del alumnado, desde el punto de vista sociológicas tales como el nivel de estudios de los padres; ocupación laboral de los padres; lugar de residencia familiar; lugar de residencia durante el curso; lugar de estudio; factores personales de género; psicopedagógicas relacionadas con los diferentes estudios de acceso; calificaciones en los estudios de bachillerato; calificación de ingreso en la Universidad; adecuación a la opción; razones para elegir la carrera; necesidades de orientación; hábitos de estudio; actitudes hacia el estudio. En cuanto a las condiciones institucionales para la docencia, según Sovero (2008), se especifican los centros de estudios; la titulación seguida; el tipo de centro; los tipos de estudios; y el curso.

Para las variables de proceso, especifico las de situación personal para la tarea académica, tales como la situación laboral del alumno y asistencia a clase. Las opiniones de los alumnos sobre las causas del bajo rendimiento. Las opiniones de los alumnos sobre el desarrollo de las clases, entre estos los programas y contenidos; 
metodología didáctica recursos; relaciones profesor-alumno; evaluación (tipos, ajuste a objetivos, instrumentos); valoración global. Tambien considera las opiniones de los profesores sobre las causas del bajo rendimiento, de los profesores sobre el desarrollo de las clases y las condiciones familiares de estudio del alumno en términos de las condiciones familiares para el estudio y la preocupación de los padres por sus estudios. Para las variables de producto, el autor refirió a los datos por alumnos, en la que considero la satisfacción con variables institucionales referidas a las condiciones de la actividad académica, profesores, compañeros de clase, carrera elegida, enseñanza que recibe, entre otras; tasas de abandono, cambio y retraso en los estudios; calificaciones en las distintas convocatorias de los distintos cursos; aprobados, presentados y matriculados en cada una de las convocatorias. Tambien mencionan los datos por cursos, centros, titulaciones y tipos de carreras.

Por otro lado, es importante señalar los actores obstaculizadores y/o facilitadores del rendimiento académico, dentro de estos factores se requieren atender algunos atributos personales, como integridad, responsabilidad, madurez, entre otros, para garantizar formar un profesional competente. Estos atributos, sin embargo, no están presentes en todos aquellos que logran el ingreso al sistema de educación superior. Por ello, para enfrentar estos desafíos, explica Aranceli y col (2006), relacionados con episodios de estrés depende de las estrategias o recursos personales de que disponen, como también del apoyo social y del ambiente académico en que se insertan. El resultado de estas interacciones puede ejercer diversos efectos en el aprendizaje (Martín, 2007). Por otra parte, también destacan factores de riesgo y factores protectores del rendimiento académico. Entre los primeros señalan como predominantes la falta de destrezas sociales y pertenencia al grupo, baja autoestima, consumo de sustancias adictivas, conducta antisocial y situación familiar irregular. Los factores protectores más frecuentes son: percepción de competencia personal, metas y expectativas futuras, afrontamiento activo a situaciones imprevistas, relaciones 
positivas con su familia, ausencia de conductas de riesgo social y maltrato familiar (Gargallo et al, 2012).

\section{METODOLOGÍA}

La metodología que siguió la investigación fue de tipo descriptiva y de campo que permitió analizar la estructura social en el rendimiento académico de los nuevos ingresos al sector universitario. En este sentido, Hernández y col (2014), considera las investigaciones descriptivas como aquellas que solo describen los hechos tal cual como se presenten sin modificar ni alterar su naturaleza. Asimismo, la investigación fue de campo, puesto que los datos fueron tomados directamente de la realidad de donde ocurren los hechos, en concordancia con lo reseñado por Arias (2012). El diseño de la investigación fue de tipo no experimental y transversal, debido a que no se manipularon las variables en estudio, sólo se detallaron los hechos del fenómeno observado tal cual como se presentaron y en un solo espacio de tiempo. Para esta investigación se seleccionó como unidades de análisis a los estudiantes que ingresaron durante los lapsos académicos del primer período de 2018.

Asimismo, se utilizó un muestreo mixto, combinando estrategias probabilísticas y no probabilísticas. Se utilizó como técnica la encuesta para la recolección de datos, la cual se concibe como el procedimiento o forma particular mediante el cual se obtiene la información para comprobar o negar las ideas iniciales que permiten establecer las conclusiones de un estudio. Hurtado (2012) expresa que "un instrumento de recolección de datos es cualquier recurso, dispositivo o formato (en papel o digital), que se utiliza para obtener, registrar o almacenar información" (p.69). En tal sentido, para efectos de este estudio se creó un instrumento tipo cuestionario para medir la variable rendimiento académico desde una perspectiva holística de su estructura social con una escala tipo Likert y cinco alternativas de respuesta a saber: Nunca, Casi nunca, A veces, Casi siempre y Siempre. 


\section{RESULTADOS}

Posterior análisis estadístico e interpretación de los resultados se pudo constatar en relación a los factores demográficos y factores pedagógicos, cognoscitivos y actitudinales lo siguiente:

\section{En cuanto a los factores demográficos}

Al interpretar los datos demográficos vinculados a la población objeto de estudio, específicamente respecto a la edad, sexo, financiación de estudios, experiencia laboral, lugar de procedencia del estudiante, estado civil, promedio de bachillerato, carrera que cursa, satisfacción por la carrera, aporte de recursos familiares y esfuerzo académico, se logró caracterizar los factores demográficos asociados al rendimiento académico en los estudiantes de nuevo ingreso, bajo las siguientes resultados:

El $67 \%$ de los sujetos encuestados corresponde al sexo femenino, mientras que el $33 \%$ corresponde al sexo masculino, encontrándose mayor concentración del sexo masculino en carreras de las ciencias duras. Asimismo el $62 \%$ de los estudiantes encuestados se encuentran en un rango entre los 19 a 26 años de edad y el porcentaje restante entre 16 y 18 años. De ello se pudo determinar que el promedio de edades se ubica entre 17 y 22 años.

La mayoría de los encuestados en un $89 \%$ son solteros, sólo un $11 \%$ están casados 0 viven en concubinato. Los hallazgos encontrados apuntan a que los encuestados considerados evolutivamente como adultos jóvenes tienen en su proyecto de vida formarse académicamente antes de iniciar una vida de pareja. Por otro lado, se constató que el $72 \%$ de los estudiantes encuestados no trabaja. Mientras que un $8 \%$ cumple con una actividad laboral y el 10 realiza labores ocasionales. Estos resultados 
reflejan la prioridad de los estudios en opinion de los investigados. El $56 \%$ de los estudiantes provienen de estudiar en instituciones privadas y el $44 \%$ de instituciones fiscales, lo que hace inferir que su nivel socio-económico es estable facilitándoles contar con el apoyo para continuar estudios a nivel universitario.

El 65\% de los estudiantes explorados tienen un promedio de notas de bachillerato entre 7 y 9 puntos, mientras que el 35\% se ubican en una promedio de notas entre 5 y 6 puntos. Es notorio que el nivel académico de ingreso a la educación universitaria es alto, lo cual podría ser un indicador predictivo de desempeño académico. Asimismo, el $86 \%$ de los estudiantes encuestados perciben que su familia se siente satisfecha con la elección de la carrera de sus hijos. Sólo un 14\% manifestó insatisfacción.

El aspecto referido a si cuentan con libros requeridos para su consulta continua, el 20\% manifestó contar con ellos, seguidamente otro 25\% refirió contar con equipos tecnológicos, en cuanto a contar con internet, un $27 \%$ lo posee como una herramienta de aprendizaje. Y un 28\% manifestó contar con todos los recursos requeridos para sus procesos de estudios. Por otro lado, el $92 \%$ de la muestra en estudio considera que su familia reconoce y valora los esfuerzos académicos que realizan los estudiantes y un $8 \%$ manifestó no contar con el reconocimiento de su familia en cuanto a los esfuerzos académicos que estos jóvenes realizan

Por consiguiente, los factores demográficos asociados al rendimiento académico constituyen elementos fundamentales que pueden contribuir o afectar las expectativas de logros educativos de los estudiantes universitarios.

\section{En relación a los factores pedagógicos, cognoscitivos y actitudinales}

La interpretación y caracterización de los resultados del análisis de los datos se presenta a continuación:

El 35\% de los estudiantes se encuentran en un nivel bajo, y el 22\% en un nivel alto. En los indicadores a nivel medio/moderado se observan: el clima de clases $(72 \%$,$) la$ integración al ambiente universitario (61\%), los hábitos de estudio (43\%) y el tamaño 
del grupo con el $42 \%$. Para los indicadores de categoría media: capacidades y habilidades (57\%); en categoría alta los estilos cognitivos (61\%). Los resultados comprueban que los estudiantes también están conscientes de la relación que existe entre el rendimiento académico de la básica superior y el de la universidad.

Por otra parte, el autoconcepto con el $71 \%$, la satisfacción con el $73 \%$, la responsabilidad hacia los aprendizajes con el $62 \%$, la planeación del futuro con el $49 \%$, y la toma de decisión ante los estudios con el $43 \%$. Asimismo, en una categoría media de interés por el estudio con el $67 \%$ y las habilidades sociales (52\%), mientras que la planeación del futuro posee el puntaje $4 \%$ ubicándose en la categoría muy bajo. El resto de los indicadores se ubican en la categoría baja.

Al analizar cada factor por separado, se observa que los factores cognoscitivos muestran porcentajes mayores que los pedagógicos y actitudinales. Mientras que los factores actitudinales reflejan la categoría más baja entre los tres factores.

Los factores cognoscitivos muestran porcentajes mayores que los pedagógicos y actitudinales. Mientras que los factores actitudinales reflejan la categoría más baja entre los tres factores. Por ello, es necesario que los factores del rendimiento académico en estudiantes universitarios deben ser vistos de una perspectiva holística integral que implique una pedagogía emergente e incluya una nueva ciencia y una nueva espiritualidad, basadas en la formación del ser y las competencias dentro del campo profesional.

\section{CONCLUSIONES}

Los factores estudiados representan los puntos débiles y las fortalezas en el rendimiento académico de los estudiantes de recién ingresos a la universidad, todo si consideramos que el rendimiento académico no sólo se da en términos de producto llamado calificaciones numéricas, sino acentuando el valor trascendental de cómo se sienten los estudiantes con su realidad universitaria, cómo afrontan las adversidades, 
cómo manejan los cambios, cómo ocurre el proceso de adaptación y su motivación académica.

Por otro lado, cabe señalar que el rendimiento académico es producto de la suma de diferentes y complejos factores que actúan en la persona que aprende, es decir en este caso en estudiantes de recién ingreso a las aulas universitarias, el cual se ve reflejado con un valor atribuido al logro del estudiante en las tareas académicas, es decir mediante las calificaciones obtenidas, con una valoración cuantitativa, cuyos resultados muestran las materias ganadas o perdidas, la deserción y el grado de éxito académico.

Asi mismo, se pudo constar que un ambiente familiar marcado por el compromiso, así como una convivencia democrática entre padres e hijos, incide en un adecuado desempeño académico de sus hijos, en tanto que el entorno de la familia ocupa un lugar muy importante. La influencia del padre o de la madre, o del adulto responsable del estudiante, influye significativamente en la vida del estudiante.

Finalmente, cabe destacar que el rendimiento académico es un indicador de eficacia y calidad educativa. De allí que estudiantes con calificaciones baja pueden asociarse a deficiencias del mismo sistema universitario y toca buscar las causas más allá que del mismo estudiante, entendiendo que sobre él no deben caer todas responsabilidades tal como se demostró en los resultados obtenidos en el estudio.

\section{REFERENCIAS CONSULTADAS}

1. Aranceli S, Perea P, Ormeño R. Evaluación de niveles, situaciones generadoras y manifestaciones de estrés académico en alumnos de tercer y cuarto año de una Facultad de Estomatología. Rev Estomatol Herediana. 2006; 16 (1):15-20.

2. Artunduaga, Martha (2008). Variables que influyen en el rendimiento académico en la universidad. En línea. Disponible en http:// www.slideshare.net/1234509876/variables-del-rendimientoacadmicouniversidad 
3. Castellanos M, Guarnizo C, y Salamanca, Y (2011). . Relación entre niveles de ansiedad y estrategias de afrontamiento en practicantes de psicología de una universidad colombiana. International Journal of Psychological Research. 2011; 4(1): 50-57.

4. Chavez Uribe A. Bienestar Psicológico y su influencia en el rendimiento académico de estudiantes de nivel medio superior. Tesis de Maestría 2006. Facultad de Psicología, Universidad de Colima, México.

5. Ezcurra A. Abandono estudiantil en educación superior. Hipótesis y conceptos. En Gluz N. (Edit.) Admisión a la universidad y selectividad social. Cuando la democratización es más que un problema de "ingresos" (pp. 23-62). Los Polvorines: Universidad Nacional de General Sarmiento. 2011.

6. Gargallo B, Suárez J, García E, Ice P, Sahuquillo M. Autoconcepto en estudiantes universitarios excelentes y en estudiantes medios. RIEOIE. 2012; 60:1-13.

7. Hernández, Roberto; Fernández, Carlos; Baptista, Pilar (2014). Metodología de la investigación. Editorial McGraw-Hill. Gta edición. México.

8. Hurtado J (2012). "El proyecto de investigación Comprensión holística de la metodología y la investigación". 7ma Edición. Quirón.

9. Luque, E y Sequi, J. (2011). Modelo teórico para la determinación del rendimiento académico general del alumno, en la enseñanza superior. Congreso Regional de Ciencia y Tecnología

10. Martín I. Estrés académico en estudiantes universitarios. Apuntes de Psicología. 2007; 25(1):87-99.

11. Maya, E., Aldana Zavala, J., \& Isea Argüelles, J. (2019). Liderazgo Directivo y Educación de Calidad. CIENCIAMATRIA, 5(9), 114 - 129. https://doi.org/10.35381/cm.v5i9.102

12. Romero, S., \& Villasmil, J. (2018). LA MADUREZ VOCACIONAL COMO FACTOR DETERMINANTE EN EL DESEMPEÑO ACADĖMICO DE LOS ESTUDIANTES DEL TERCER SEMESTRE DE MEDICINA DE LA MODALIDAD ADI DE LA UNIVERSIDAD NACIONAL EXPERIMENTAL FRANCISCO DE MIRANDA. Revista Arbitrada Interdisciplinaria Koinonía, 2(4), 148-179. Recuperado de 


\section{http://fundacionkoinonia.com.ve/ojs/index.php/revistakoinonia/article/view/131/11} 1

13. Silva P. La universidad Latinoamericana en la época actual. Revista Cursos precongreso Universidad 2010. La Habana. Editorial Universitaria. 2010. 54 p.

14. Sovero, Guillermo (2008), El Rendimiento Académico Factor determinante del Aprendizaje. Arequipa - Perú. Ed. Edimag.

15. Tejedor, F; García, A- Varcarcel (2008) Causas del bajo rendimiento académico del estudiante universitario. Revista de Educación, Universidad de Salamanca, p 443-473

16. Vargas Díez, J. (2007). Factores diferenciales del rendimiento académico en educación superior. Tesis doctoral, Departamento MIDE, Universidad Complutense de Madrid,

17. Velásquez C, Montgomery W, Montero V, Pomalaya R, Dioses A, Velásquez N, Araki R y Reynoso D. (2008). Bienestar psicológico, asertividad y rendimiento académico en estudiantes universitarios sanmarquinos. Rev Investigación Psicológica. 11(2):139-152.

\section{REFERENCES CONSULTED}

1. Aranceli S, Perea P, Ormeño R. Evaluation of levels, generating situations and manifestations of academic stress in third and fourth year students of a Stomatology Faculty. Rev Estomatol Herediana. 2006; 16 (1): 15-20.

2. Artunduaga, Martha (2008). Variables that influence academic performance in the university. Online. Available at http: // www.slideshare.net/1234509876/variablesdel-rendimientoacadmico-universidad

3. Castellanos M, Guarnizo C, and Salamanca, Y (2011). . Relationship between anxiety levels and coping strategies in psychology practitioners of a Colombian university. International Journal of Psychological Research. 2011; 4 (1): 50-57.

4. Chavez Uribe A. Psychological Wellbeing and its influence on the academic performance of upper middle level students. Master thesis 2006. Faculty of Psychology, University of Colima, Mexico. 
5. Ezcurra A. Student dropout in higher education. Hypothesis and concepts. In Gluz N. (Edit.) Admission to university and social selectivity. When democratization is more than a problem of "income" (pp. 23-62). Los Polvorines: National University of General Sarmiento. 2011

6. Gargallo B, Suárez J, García E, Ice P, Sahuquillo M. Self-concept in excellent university students and in middle students. RIEOIE 2012; 60: 1-13.

7. Hernández, Roberto; Fernández, Carlos; Baptista, Pilar (2014). Investigation methodology. McGraw-Hill editorial. 6th edition. Mexico.

8. Hurtado J (2012). "The research project Holistic understanding of methodology and research". 7th Edition. Chiron

9. Luque, E and Sequi, J. (2011). Theoretical model for the determination of the student's overall academic performance, in higher education. Regional Congress of Science and Technology

10. Martín I. Academic stress in university students. Psychology Notes. 2007; 25 (1): 87-99.

11. Maya, E., Aldana Zavala, J., \& Isea Argüelles, J. (2019). Leadership Leadership and Quality Education. CIENCIAMATRIA, 5 (9), 114-129. Https://doi.org/10.35381/cm.v5i9.102

12. Romero, S., \& Villasmil, J. (2018). VOCATIONAL MATURITY AS A DETERMINING FACTOR IN THE ACADĖMIC PERFORMANCE OF STUDENTS OF THE THIRD SEMESTER OF MEDICINE OF THE ADI MODALITY OF THE FRANCISCO MIRANDA NATIONAL EXPERIMENTAL UNIVERSITY. Interdisciplinary Arbitrated Review Koinonía, 2 (4), 148-179. Retrieved from http://fundacionkoinonia.com.ve/ojs/index.php/revistakoinonia/article/view/131/11 1

13. Silva P. The Latin American university at the present time. Magazine Pre-congress courses University 2010. Havana. University Publishing. 2010. 54 p.

14. Sovero, Guillermo (2008), The Academic Performance Determining Factor of Learning. Arequipa, Peru. Ed. Edimag.

15. Weaver, F; García, A- Varcarcel (2008) Causes of the low academic performance of the university student. Education Magazine, University of Salamanca, p 443-473 


\section{CIENCIAMATRIA}

Revista Interdisciplinaria de Humanidades, Educación, Ciencia y Tecnología

Año VI. Vol. VI. No10. Enero - Julio 2020

Hecho el depósito de ley: pp201602FA4721

ISSN-L: 2542-3029; ISSN: 2610-802X

Universidad Nacional Experimental Francisco de Miranda (UNEFM). Santa Ana de Coro. Venezuela

Gabriela Esperanza Zambrano Vera; María Antonieta Palma Cedeño; Silvia Monserrate Suástegui Solórzano

16. Vargas Díez, J. (2007). Differential factors of academic performance in higher education. Doctoral thesis, MIDE Department, Complutense University of Madrid,

17. Velásquez C, Montgomery W, Montero V, Pomalaya R, Dioses A, Velásquez $\mathrm{N}$, Araki R and Reynoso D. (2008). Psychological well-being, assertiveness and academic performance in Sanmarquinos university students. Rev Psychological Research. 11 (2): 139-152.

C2020 por los autores. Este artículo es de acceso abierto y distribuido según los términos y condiciones de la licencia Creative Commons Atribución-NoComercial-Compartirlgual 4.0 Internacional (CC BY-NC-SA 4.0)

(https://creativecommons.org/licenses/by-nc-sa/4.0/). 